\title{
Stroke Leads to Abnormal Coordination of the Multiple Upper Extremity Joints During Passive Movement
}

\section{Kyung Koh}

University of Maryland baltimore https://orcid.org/0000-0003-3481-4152

\section{Dongwon Kim}

University of Maryland School of Medicine

Giovanni Oppizzi

University of Maryland at College Park

Chunyang Zhang

University of Maryland School of Medicine

Glenn Kehs

University of Maryland School of Medicine

Li-Qun Zhang ( $\nabla$ l-zhang@som.umaryland.edu )

University of Maryland, Baltimore https://orcid.org/0000-0001-5882-7965

\section{Research}

Keywords: upper extremity, rehabilitation, kinematic , exoskeleton

Posted Date: September 14th, 2020

DOI: https://doi.org/10.21203/rs.3.rs-72253/v1

License: () (1) This work is licensed under a Creative Commons Attribution 4.0 International License. Read Full License 
Stroke leads to abnormal coordination of the multiple upper extremity joints during passive movement

Kyung $\mathrm{Koh}^{1}$, Dongwon Kim ${ }^{1,4}$, Giovanni Oppizzi ${ }^{4}$, Chunyang Zhang ${ }^{1}$, Glenn Kehs ${ }^{2}$, Li-Qun Zhang ${ }^{1,3-4 *}$

${ }^{1}$ Department of Physical Therapy \& Rehabilitation Science, University of Maryland, Baltimore, MD 21201

${ }^{2}$ University of Maryland Rehabilitation and Orthopaedic Institute, Baltimore, MD

${ }^{3}$ Department of Orthopaedics, University of Maryland, Baltimore, MD 21201

${ }^{4}$ Fischell Department of Bioengineering, University of Maryland, College Park, MD 20742

Correspondence:

Li-Qun Zhang, $\mathrm{PhD}$

Department of Physical Therapy \& Rehabilitation Science

University of Maryland, Baltimore

100 Penn St, Baltimore, MD 21201

1-zhang@som.umaryland.edu 


\begin{abstract}
Background

Motor impairments in the upper extremity (UE) is one of the most common deficits after stroke. Even though understanding of UE coordination deficits in persons with strokes is critical for better identification of motor impairment and planning for rehabilitation, it is still not clear how stroke affects coordination patterns of multi-joint movements in the UE. Here, we investigated kinematic and kinetic coordination patterns of UE after stroke during controlled passive arm movement.
\end{abstract}

Methods

An exoskeleton multi-joint robot moved the participant's arm in the horizontal plane back and forth in 8 repetitions, in inward movement (i.e. toward the body) and outward movement (i.e. away from the body). The uncontrolled manifold analysis (UCM) was used to quantify kinematic and kinetic coordination patterns of the UE. Variability of joint angles and torques were decomposed into task-relevant variability $(T R V)$ and task-irrelevant variability $(T I V)$. An index of coordination (IC) was defined based on $T R V$ and $T I V$.

Results

We found that the $I C$ of joint torques in the stroke group significantly decreased during outward movement in comparison to that during inward movement, while $I C$ of the control group showed no difference between the two movement directions. The decreased $I C$ in the stroke group during outward movement was mainly due to the increased $T R V$ of joint torques. In the further analysis of individual joint level, during outward movement, stroke group had a greater $T R V$ of joint torques at all joints while during inward movement, stroke group had a lower $T R V$ of joint torques at elbow joint. 


\section{Conclusions}

Our results indicate that the stroke can cause the kinetic coordination deficits induced during a passive movement especially in outward movement. Our findings suggest that it is important to consider the passive kinetic coordination deficits to enhance post-stroke rehabilitation interventions.

Trial registration

clinicaltrials.gov, ID: NCT02359812. Registered 23 January 2015; Last Updated 06 August 2020. 


\section{Background}

About 795,000 people suffer strokes every year in the United States and stroke is the third leading cause of death. Stroke survivors often live with long-term motor functional impairments in daily activities [1]. Motor impairment in upper extremity (UE) is the most common deficit after stroke, reported in more than $80 \%$ of acute and $40 \%$ of chronic stroke survivors [2]. Even though there are several researches conducted to improve motor functions in stroke survivors by relearning and developing compensatory mechanisms, their impaired UE function for activities of daily living is still regarded as one of most challenging sequelae of a strokes [3-5].

It has been reported that after neurological deficit such as stroke, coordination patterns of UE movement are altered [6-8]. Clinically, the term synergy has been used to describe coordination deficits evident in abnormal co-activation of muscles after stroke. Abnormal synergies after stroke involve a tight coupling, respectively[9]. Such abnormal couplings have been observed in previous studies of UE kinetics reporting abnormal torque patterns between elbow extension and shoulder adduction in isometric maximum voluntary torque [10, 11]. However, these studies provide little information about the underlying mechanics to reveal how the central nervous system (CNS) generates abnormal coordination patterns of UE movements.

In recent years, the concept of synergy has emerged in neuroscience and motor control as a fundamental mechanism of the CNS to control multiple degree of freedom of the motor apparatus for desired inter-joint coordination. Motor synergy has been examined under the framework of Uncontrolled Manifold analysis[12, 13]. The UCM analysis provides a quantitative approach of quantifying how multi-joint movements are organized or controlled by examining the structure of variability in the elemental variables (e.g. arm joint angles) with 
respect to task variables (e.g. hand position Cartesian coordinates). In the arm reaching task, for example, the UCM quantifies the variance across trials of joint combinations partitioned into two components at every point in the hand's trajectory; joint variance that led to a consistent hand position (i.e. $T I V$, task-irrelevant variability) and joint variance that led to an inconsistent hand position from trial to trial (i.e. $T R V$, task-relevant variability). The UCM approach has been applied to several voluntary motor tasks. In healthy populations, many studies found that $T I V$ was greater than $T R V$, indicating more flexibility in utilizing degree of freedoms (DoF) to accomplish a task. In a person with neurological diseases such as Parkinson's disease [14], olivoponto-cerebellar atrophy [15], multiple sclerosis [16] and spinocerebellar degeneration [17], different patterns of motor synergies have been observed often with decreased $T I V$ and increased $T R V$ as compared to healthy groups. However, previous stroke studies have reported inconsistent results on $T R V$ and $T I V$ between stroke group and healthy group during a UE reaching task [1820]. One of the possibilities for the inconsistent results may be attributed to changes in passive properties of UE after stroke. Several previous studies have found increased non-reflex passive joint stiffness in subjects with chronic stroke [21, 22]. Such an increased stiffness may be reflected in formation of abnormal variability of UE.

Recently, advanced robotic technologies have made it possible to systematically investigate and assess multi-joint movements [23]. In our previous work [24], a multi-joint intelligent rehabilitation robot, named IntelliArm, was developed to control the shoulder, elbow and wrist individually and simultaneously. IntelliArm allows us to evaluate more quantitative and systematic characterizations of neuro-muscular changes across multiple joints. Here, using IntelliArm, we investigated how stroke affect UE coordination patterns during passive movement. Hypothesis is a two-fold: 1) abnormal coordination patterns would be more severe during 
outward movement (i.e. away from the body) as compared to inward movement (i.e. toward the body) due to stroke accompanied by flexor hypertonia of UE and 2) wrist joint would contribute most to the abnormal coordination patterns, based on the classical perception of a proximal to distal gradient of motor deficits in UE after stroke (i.e., distal segment is more severe than proximal segment) $[9,25]$.

\section{Methods}

\section{Participants}

10 stroke survivors and 10 age-matched control subjects were recruited for this study. All the control subjects were right-handed ( 8 males and 2 females, mean age 50 years, SD 12.4). Three stroke survivors were right hemiparetic, and the other seven patients were left hemiparetic ( 8 males and 2 females, mean age 62 years, SD 8.4). Inclusion criteria of the stroke patients were a stable medical condition, an interval of at least 1 year since stroke onset with cognitive ability to follow simple instructions. Prior to the experiment, each participant gave a written consent approved by the institutional review board of University of Maryland, Baltimore.

\section{Experiments}

In order to assess UE function, we used a rehabilitation robot for neurorehabilitation of the shoulder elbow, and wrist joints we developed previously (Fig. 1a). The robot called IntelliArm was designed to perform a two-dimensional motion on the horizontal plane with gravity support of UE. All subjects were asked to sit upright comfortably, and to put the upper arm, forearm and hand on IntelliArm (Fig. 1a). The robot arm lengths for the upper arm and forearm were then adjusted to align mechanical axes for shoulder horizontal adduction/abduction, and elbow and wrist flexion/extension with the corresponding subject's anatomical joint axes. 
The subject's upper arm and forearm were strapped to the corresponding braces to ensure wellalignment throughout the experiment. Initial position was at shoulder horizontal adduction of $70^{\circ}$, elbow flexion of $60^{\circ}$ and wrist flexion of $0^{\circ}$, respectively (Fig. 1b).

IntelliArm produces simultaneous movements at shoulder, elbow, and wrist joints, starting at initial position to outward direction followed by inward direction. Each joint was moved until it reaches pre-specified limits for either the joint resistance torque or angular position [26]. Torque and angular position limits were set to be $\pm 3 \mathrm{~N} \cdot \mathrm{m}$ and $0^{\circ}$ to $120^{\circ}$ for shoulder horizontal adduction/abduction, $\pm 2 \mathrm{~N} \cdot \mathrm{m}$ and $10^{\circ}$ to $90^{\circ}$ for elbow flexion/extension, and $\pm 1.5 \mathrm{~N} \cdot \mathrm{m}$ and $-45^{\circ}$ to $45^{\circ}$ for wrist flexion/extension, respectively. There was 3 seconds holding time at initial position and all movements were performed for 8 repetitions (Fig. 2). Joint torques and angles were recorded simultaneously using a torque sensor and motor encoder, respectively. Each joint was moved at slow speed of $10^{\circ} / \mathrm{s}$ was selected in order to minimize reflex mediated actions and manifest passive mechanical properties [22].

\section{$\underline{\text { Uncontrolled manifold analysis }}$}

The UCM analysis has been used to assess multi-joint coordination patterns in a redundant system by quantifying variability of elemental variables (i.e., joint angles or torques) with respect to changes in performance variable (i.e. position or force or the tip of the endeffector) [27]. Using the UCM analysis, we examined how multi-joints interact each other to stabilize the actions of the end-effector in terms of kinematics and kinetics.

\section{$\underline{\text { Kinematics }}$}

In order to quantify coordination patterns of multi-joint angles, the 2-D position of the tip of the end-effector (i.e., the tip of the index finger or the tip of the rod) was selected as 
performance variable, whereas joint-angles were selected as elemental variables. First, a forward-kinematics model in horizontal plane movement was created for the human arm as follows:

$$
\begin{gathered}
{\left[\begin{array}{l}
P_{x} \\
P_{y}
\end{array}\right]=\left[\begin{array}{l}
f_{1}\left(\theta_{s}, \theta_{e}, \theta_{w}\right) \\
f_{2}\left(\theta_{s}, \theta_{e}, \theta_{w}\right)
\end{array}\right]} \\
f_{1}=l_{s} \cos \left(\theta_{s}\right)+l_{s} \cos \left(\theta_{e}\right)+l_{s} \cos \left(\theta_{w}\right) \\
f_{2}=l_{S} \sin \left(\theta_{s}\right)+l_{S} \sin \left(\theta_{e}\right)+l_{s} \sin \left(\theta_{w}\right)
\end{gathered}
$$

where the three joints rotations are shoulder horizontal abduction-adduction $\left(\theta_{s}\right)$, elbow flexion-extension $\left(\theta_{e}\right)$, and wrist flexion-extension $\left(\theta_{w}\right)$, and the segment lengths are $l_{s}, l_{e}$ and $l_{w}$ for shoulder, elbow and wrist, respectively.

Using the Jacobian matrix (J), a linearized task equation, position of the end-effector as a function of joint angles was computed.

$$
\left[\begin{array}{l}
\partial P_{x} \\
\partial P_{y}
\end{array}\right]=\left[\begin{array}{lll}
\frac{\partial f_{1}}{\partial \theta_{s}} & \frac{\partial f_{1}}{\partial \theta_{e}} & \frac{\partial f_{1}}{\partial \theta_{w}} \\
\frac{\partial f_{2}}{\partial \theta_{s}} & \frac{\partial f_{2}}{\partial \theta_{e}} & \frac{\partial f_{2}}{\partial \theta_{w}}
\end{array}\right]\left[\begin{array}{l}
\partial \theta_{s} \\
\partial \theta_{e} \\
\partial \theta_{w}
\end{array}\right]
$$

The null space of $\mathbf{J}$ represents the changes in elemental variables that do not lead to a change in the performance variable, referred to as task-irrelevant space, whereas the orthogonal component of $\mathrm{J}$ represents the changes in elemental variables that do lead to a change in the performance variable, referred to as task-relevant space. The basis vectors, $\varepsilon$, for the null space was calculated using Matlab null() function such that $J \varepsilon=0$. 
Two types of variability, the deviations from the average trajectories in joint space projected onto task-irrelevant space, $\theta_{T I R}$, and task-relevant space, $\theta_{T R}$, respectively were computed as follows.

$$
\begin{gathered}
\theta_{T I R}=\varepsilon \varepsilon^{T}(\theta-\bar{\theta}) \\
\theta_{T R}=(\theta-\bar{\theta})-\theta_{T I R}
\end{gathered}
$$

We calculated variance of $\theta_{T I R}$ and $\theta_{T R}$ to measure $T I V$ and $T R V$ of joint angles, respectively.

$$
T I V_{\theta}=\frac{1}{n} \sum_{i=1}^{n}\left|\theta_{T I R}\right|^{2}
$$

and

$$
T R V_{\theta}=\frac{1}{n} \sum_{i=1}^{n}\left|\theta_{T R}\right|^{2}
$$

where $n$ is a number of trails and here $n=8$.

Note that $T I V_{\theta}$ and $T R V_{\theta}$ were transformed to correct for a non-normal distribution using the $\log$ transformation [28].

$\underline{\text { Kinetics }}$

Similar to quantification of kinematic coordination, we quantified kinetic coordination patterns of UE. Using the Jacobian matrix calculated, a linearized task equation, force of the endeffector as a function of joint torques was computed.

$$
\left[\begin{array}{l}
F_{x} \\
F_{y}
\end{array}\right]=J^{+}\left[\begin{array}{c}
\tau_{s} \\
\tau_{e} \\
\tau_{w}
\end{array}\right]
$$

where $J^{+}$is a pseudoinverse, $J^{+}=\left(J \cdot J^{T}\right)^{-1} \cdot J$. 
The basis vectors, $\varepsilon$, for the null space of $J^{+}$was calculated such that $J^{+} \varepsilon=0$.

The variabilities, the deviations from the average trajectories in joint space are projected onto task-irrelevant space, $\tau_{T I R}$, and task-relevant space, $\tau_{T R}$, respectively.

$$
\begin{gathered}
\tau_{T I R}=\varepsilon \varepsilon^{T}(\tau-\bar{\tau}) \\
\tau_{T R}=(\tau-\bar{\tau})-\tau_{T I R}
\end{gathered}
$$

The variance per joint of the projected deviations is

$$
T I V_{\tau}=\frac{1}{n \cdot d_{T I R}} \sum_{i=1}^{n}\left|\tau_{T I R}\right|^{2}
$$

and

$$
T R V_{\tau}=\frac{1}{n \cdot d_{T I R}} \sum_{i=1}^{n}\left|\tau_{T R}\right|^{2}
$$

where $d_{T I R}$ and $d_{T R}$ are dimensions of task-irrelevant space, $d_{T I R}=d_{\text {joint }}-d_{\text {task }}$, and task-relevant space, $d_{T R}=d_{\text {joint }}-d_{T I R}$, respectively. $d_{\text {joint }}$ and $d_{\text {task }}$ are a number of joints $(=3)$ and a dimension of a task space $(=2)$, respectively.

Note that $T I V_{\tau}$ and $T R V_{\tau}$ were transformed to correct for a non-normal distribution using the $\log$ transformation [28].

An index of coordination $(I C)$, the ratio between these two variances, quantifies to what extent the joint variables are coordinated to keep the target trajectory in task space.

$$
I C=\frac{T I V-T R V}{T I V+T R V}
$$

Importantly, this ratio is not necessarily related to the overall joint-space variance; instead, it quantifies its structure with respect to the task space. So far, during an active movement, IC has been used to investigate the CNS's control mechanisms about the extent of 
stabilization of the particular task variable. When $I C$ is larger than zero, a higher $I C$ indicates that joint space variables are more coordinated such that the task variable is stabilized. If $I C$ is equal to or smaller than zero, the coordination in joint space is indifferent to the particular task variable, or even destabilizes it.

\section{$\underline{\text { Statistical analysis }}$}

A three-way repeated measures ANOVA with factors Joint (3 levels: shoulder, elbow, and wrist), Direction (2 levels: inward and outward) and Group (2 levels: stroke and control) were used to test the differences between conditions. In addition to this, a two-way repeated measures ANOVA with factors Direction and Group was performed to test differences of index of coordination. The level of statistical significance was set at $p=0.05$. A post-hoc test was performed where necessary.

\section{Results}

\section{Index of Coordination}

$I C$ of joint angles, $T R V_{\theta}$ and $T I V_{\theta}$ during inward and outward movements were depicted in Figure 3. In both $T R V_{\theta}$ and $T I V_{\theta}$, there was a significant main effect of $\operatorname{Group}\left(T R V_{\theta}: \mathrm{F}_{1,17}=\right.$ 20.657; $\mathrm{p}<0.001$, and $T I V_{\theta}: \mathrm{F}_{1,17}=13.760 ; \mathrm{p}=0.002$ ), indicating Stroke group had significantly higher $T R V_{\theta}$ and $T I V_{\theta}$ than Control group. However, there was no significant difference on $I C$ of joint angles between Stroke group and Control group, which were supported by no significant main effects of Direction $\left(\mathrm{F}_{1,17}=0.868 ; \mathrm{p}=0.364\right)$ and Group $\left(\mathrm{F}_{1,17}=0.246 ; \mathrm{p}=0.626\right)$, along with no significant interaction Direction $\times$ Group $\left(\mathrm{F}_{1,17}=0.078 ; \mathrm{p}=0.783\right)$. 
In terms of joint torque $I C$ depicted in Figure 4a, ANOVA with repeated measures revealed that a significant main effect of Direction $\left(\mathrm{F}_{1,17}=7.345 ; \mathrm{p}=0.014\right)$ and interaction Direction $\times$ Group $\left(\mathrm{F}_{1,17}=6.079 ; \mathrm{p}=0.024\right)$. Pairwise comparisons showed that Stroke group had a significant decrease in $I C$ during outward than during inward $(\mathrm{p}=0.002)$ while Control group had no significant difference in $I C(\mathrm{p}=0.865)$ between movement directions. Although there was a trend of difference in $I C$ between groups during inward movement, it was not statistically significant. In $T I V_{\tau}$ depicted in Figure $4 \mathrm{c}$, there was no significant main effect of Direction $\left(\mathrm{F}_{1,17}\right.$ $=0.087 ; \mathrm{p}=0.771)$, Group $\left(\mathrm{F}_{1,17}=4.077 ; \mathrm{p}=0.059\right)$, and interaction Direction $\times$ Group $\left(\right.$ TRV $_{\tau}$ : $\left.\mathrm{F}_{1,17}=1.503 ; \mathrm{p}=0.236\right)$. However, in $T R V_{\tau}$ depicted in Figure $4 \mathrm{~b}$, there was a significant main effect of Direction $\left(\mathrm{F}_{1,17}=26.686 ; \mathrm{p}<0.001\right)$, and interaction Direction $\times$ Group $\left(\mathrm{F}_{1,17}=9.830\right.$; $\mathrm{p}=0.006)$, along with no significant main effect of Group $\left(\mathrm{F}_{1,17}=0.872 ; \mathrm{p}=0.363\right)$. This result indicates that the decreased $I C$ of joint torques during outward movement was mainly attributed to the increased $T R V_{\tau}$.

\section{Individual joint contributions}

Both $T R V_{\theta}$ and $\operatorname{TIV}_{\theta}$ were decomposed into an individual joint level in order to investigate individual joint contributions. There was no significant interaction Direction $\times$ Joint $\times$ Group effect observed on both $\operatorname{TRV}_{\theta}\left(\mathrm{F}_{2,34}=0.709 ; \mathrm{p}=0.499\right)$ and $\operatorname{TIV}_{\theta}\left(\mathrm{F}_{2,34}=2.782\right.$; $\mathrm{p}=0.075)$. However, in terms of $T R V_{\tau}$ and $T I V_{\tau}$ in individual joint level shown in Figure 5, there was a significant interaction Direction $\times$ Joint $\times$ Group effect on $\operatorname{TIV}_{\tau}\left(\mathrm{F}_{2,34}=5.744 ; \mathrm{p}=0.007\right)$ and $T R V_{\tau}\left(\mathrm{F}_{2,34}=58.613 ; \mathrm{p}<0.001\right)$. Pairwise comparisons revealed that Stroke group at shoulder joint had a significant higher $T I V_{\tau}$ during inward movement $(\mathrm{p}=0.028)$ and outward movement ( $\mathrm{p}<0.001)$ as compared to Control group. Pairwise comparisons on $T R V_{\tau}$ revealed that during 
inward, Stroke group at elbow joint had a significant lower $T R V_{\tau}$ as compared to Control group ( $\mathrm{p}<0.001$ ). During outward, Stroke group at all joints had a significant lower $T R V_{\tau}$ as compared to Control group (shoulder: $\mathrm{p}=0.026$, elbow: $\mathrm{p}=0.022$, and wrist: $\mathrm{p}=0.045$ ).

\section{Discussion}

The aim of this study was to investigate how stroke affect UE coordination patterns during passive movement. Using the UCM approach, UE coordination patterns were analyzed and quantified as $I C$, a ratio of $T R V$ and $T I V$. We expected that abnormal coordination patterns would be more severe during outward movement as compared to inward movement due to stroke with flexor hypertonia. Kinematically, we found no significant coordination patterns between Stroke group and Control group. However, kinetically, we found that index of coordination, IC, in Stroke group significantly decreased during outward as compared to during inward while $I C$ in Control group remained unchanged between movement directions. Our finding of the decreased $I C$ in Stroke group during outward movement was mainly due to the increased $T R V$ of joint torques. These results indicate that during passive stretching, joint torques were generated in a way that force at the end-effector (i.e. hand) became more variable or less inconsistent during outward movement than inward movement. In other words, Stroke group had abnormal coordination patterns of joint torques during outward movements by producing joint torques that resulted in inconsistent generation of force at the hand.

Our results did not support another hypothesis that the wrist joint would contribute most to the abnormal coordination patterns based on the classical perception of a proximal to distal gradient of motor deficits in UE after stroke. The classical perception of clinicians about individuals who have recently suffered a stroke is that paresis of distal upper limb segments is 
more severe than paresis of proximal upper limb segments [9, 25]. Based on this clinical perception, it is often presumed that the loss of distal segment movement is responsible for the loss of hand function after stroke [29]. However, other studies found contradictory findings, reporting that a proximal to distal gradient of motor deficits is not present post stroke [30]. Our results in the current study in consistent with these studies suggested proximal to distal gradient of motor deficits in UE after study would not exist.

Our observations extend our understanding of stroke in the context of coordination patterns of UE movements. In the current study, it was the first attempt to use the UCM approach for coordination patterns during involuntary movements or passive stretching. So far, the UCM approach has been successful used to investigate the CNS control mechanism underlying coordinated movements during several voluntary tasks [12, 31-35]. The idea behind motor coordination in the framework of the UCM approach is that the CNS utilizes element variables in a way to ensure stability of performance variable, which reflects in task-specific pattern of distribution of the element variables, referred as to the principle of abundance [36]. In support of the principle of abundance, several studies suggested that have a greater values of $T I V$ over $T R V$ would be beneficial in dealing with unexpected perturbations [37], fatigue [38], and secondary tasks [39]. In pathological and gerontological studies [14-17], abnormal coordination patterns have been observed indicating pathological and gerontological markers of deficits or changes in the CNS control mechanisms for a redundant motor system. In the current study, however, considering that no or minimal active control by the CNS is involved during passive stretching, either positive, negative or zero of $I C$ would not indicate good or bad (stabilizing or destabilizing) coordination on the CNS's control, rather it represents passive coupling patterns of inter-joint. Any differences on $I C$ in stroke group as compared to control group may indicate that passive 
structure of abnormal coordination patterns. Our findings that stroke group had abnormal kinetic coordination across the joints, possibly tied to the increased coupling in spastic upper extremity $[21,22]$. Thus, our findings of decreased $I C$ in Stroke group during outward movements implies that increased hyperexcitability of the stretch reflex after stroke may play a role in formation of abnormal coordination patterns especially during UE outward movements.

We found that stroke group had a significant higher both on $T I V$ and $T R V$ as compared to control group. Our results provide meaningful insights into understating of coordination deficits after stroke. In previous stroke studies, coordination patterns during voluntary movements were not consistent, reporting unchanged $I C$ [18, 40], decreased $I C$ [41], and increased $I C$ [20], along with unchanged $T I V[18,40]$, and greater $T I V[19,20]$. However, $T R V$ was found to be greater than in control $[19,20,41]$. The previous studies suggested that this unexpected finding might have resulted from large inherent variability of neural signals between motor cortex and effectors [42] or being more dependency of effectors in the patients with stroke [43]. However, this finding is relatively contradictory to the principle of motor abundance because this finding may imply that stroke survivors would have superior control abilities to utilize DoFs for completion of particular tasks. In addition, based on the finding of the current study, the inconsistent findings of $I C$ in previous studies may be attributed to changes in passive coordination patterns. Thus, the findings of the current study can provide new aspects of coordination deficits and be used for better identification of abnormal coordination patterns during active movements by subtracting the passive components of abnormal coordination patterns, which warrants further investigations.

\section{Conclusions}


In summary, our findings clearly demonstrate that passive UE movements in stroke individuals induces abnormal coordination patterns especially in outward direction. Such an abnormal coordination patterns in stroke survivors was mainly due to increased variability of joint torques that led to inconsistent end-effector (or hand) force compared with healthy adults. Therefore, the abnormal coordination patterns provide important information for understanding of the CNS's control deficits following stroke.

\author{
Abbreviations \\ UE: Upper extremity \\ CNS: Central nervous system \\ UCM: Uncontrolled Manifold analysis \\ TIV: Task-irrelevant variability \\ $T R V$ : Task-relevant variability \\ DoF: Degree of freedoms \\ $I C:$ An index of coordination
}

\title{
Declarations
}

Ethics approval and consent to participate

This study was approved by the institutional review board of the University of Maryland Baltimore (IRB protocol number: HP-00074975). The study was publicly registered on clinicaltrials.gov with Clinical Trials Identifier NCT02359812. The patient and family were informed beforehand and provided consent to participate.

Consent for publication 
Written informed consent was obtained from the patient for publication of this manuscript. A copy of the written consent is available for review by the Editor of this journal.

Availability of data and materials

The datasets used and/or analyzed during the current study are available from the corresponding author on reasonable request (1-zhang@som.umaryland.edu).

\section{Competing Interests}

L.-Q. Zhang has an equity position in Rehabtek LLC, which received federal funding in developing the IntelliArm rehabilitation robot used in this study.

Funding

This research was supported in part by the National Institute on Disability, Independent Living, and Rehabilitation Research (NIDILRR, 90DP0099) and the NIH grant P30 AG028747.

Authors' contributions

Author contributions: K.K., DW. K., and LQ.Z., designed research; K.K., DW. K., and C.Z., performed the experiments; K.K., DW.K., G.O., C.Z., and LQ.Z., analysed data; K.K., DW.K., G.O., C.Z., G.K., and LQ.Z., wrote the paper.

Acknowledgements

Not applicable 


\section{Reference}

1. Mayo NE, Wood-Dauphinee S, Ahmed S, Gordon C, Higgins J, McEwen S, Salbach N: Disablement following stroke. Disabil Rehabil 1999, 21(5-6):258-268.

2. Cramer SC, Nelles G, Benson RR, Kaplan JD, Parker RA, Kwong KK, Kennedy DN, Finklestein SP, Rosen BR: A functional MRI study of subjects recovered from hemiparetic stroke. Stroke 1997, 28(12):2518-2527.

3. Wade DT, Langton-Hewer R, Wood VA, Skilbeck CE, Ismail HM: The hemiplegic arm after stroke: measurement and recovery. $J$ Neurol Neurosurg Psychiatry 1983, 46(6):521-524.

4. Sunderland A, Fletcher D, Bradley L, Tinson D, Hewer RL, Wade DT: Enhanced physical therapy for arm function after stroke: a one year follow up study. J Neurol Neurosurg Psychiatry 1994, 57(7):856-858.

5. Langhorne $P$, Coupar F, Pollock A: Motor recovery after stroke: a systematic review. Lancet Neurol 2009, 8(8):741-754.

6. Cirstea MC, Mitnitski $A B$, Feldman AG, Levin MF: Interjoint coordination dynamics during reaching in stroke. Exp Brain Res 2003, 151(3):289-300.

7. Dipietro L, Krebs HI, Fasoli SE, Volpe BT, Stein J, Bever C, Hogan N: Changing motor synergies in chronic stroke. J Neurophysiol 2007, 98(2):757-768.

8. Brunnstrom SJAna: Movement therapy in hemiplegia. 1970:113-122.

9. Twitchell TE: The restoration of motor function following hemiplegia in man. Brain 1951, 74(4):443-480.

10. Dewald JP, Beer RF: Abnormal joint torque patterns in the paretic upper limb of subjects with hemiparesis. Muscle Nerve 2001, 24(2):273-283.

11. Beer RF, Given JD, Dewald JPJAopm, rehabilitation: Task-dependent weakness at the elbow in patients with hemiparesis. 1999, 80(7):766-772.

12. Scholz JP, Reisman D, Schoner G: Effects of varying task constraints on solutions to joint coordination in a sit-to-stand task. Exp Brain Res 2001, 141(4):485-500.

13. Latash ML: Motor synergies and the equilibrium-point hypothesis. Motor Control 2010, 14(3):294-322.

14. Jo HJ, Park J, Lewis MM, Huang X, Latash ML: Prehension synergies and hand function in earlystage Parkinson's disease. Experimental brain research 2015, 233(2):425-440.

15. Park J, Lewis MM, Huang X, Latash ML: Effects of olivo-ponto-cerebellar atrophy (OPCA) on finger interaction and coordination. Clinical Neurophysiology 2013, 124(5):991-998.

16. Jo HJ, Mattos D, Lucassen EB, Huang X, Latash ML: Changes in multidigit synergies and their feed-forward adjustments in multiple sclerosis. Journal of motor behavior 2017, 49(2):218-228.

17. Asaka T, Wang Y: Feedforward postural muscle modes and multi-mode coordination in mild cerebellar ataxia. Exp Brain Res 2011, 210(1):153-163.

18. Reisman DS, Scholz JP: Aspects of joint coordination are preserved during pointing in persons with post-stroke hemiparesis. Brain : a journal of neurology 2003, 126(Pt 11):2510-2527.

19. Kang N, Cauraugh JH: Bilateral synergy as an index of force coordination in chronic stroke. Experimental brain research 2017, 235(5):1501-1509.

20. Kim Y, Kim W-S, Koh K, Yoon B, Damiano DL, Shim JK: Deficits in motor abilities for multi-finger force control in hemiparetic stroke survivors. Experimental brain research 2016, 234(8):23912402.

21. Mirbagheri MM, Alibiglou L, Thajchayapong M, Rymer WZ: Muscle and reflex changes with varying joint angle in hemiparetic stroke. Journal of neuroengineering and rehabilitation 2008, 5:6. 
22. Zhang LQ, Son J, Park HS, Kang SH, Lee Y, Ren Y: Changes of Shoulder, Elbow, and Wrist Stiffness Matrix Post Stroke. IEEE transactions on neural systems and rehabilitation engineering : a publication of the IEEE Engineering in Medicine and Biology Society 2017, 25(7):844-851.

23. Chang WH, Kim YH: Robot-assisted Therapy in Stroke Rehabilitation. Journal of stroke 2013, 15(3):174-181.

24. Park H-S, Ren Y, Zhang L-Q: IntelliArm: An exoskeleton for diagnosis and treatment of patients with neurological impairments. In: 2008 2nd IEEE RAS \& EMBS International Conference on Biomedical Robotics and Biomechatronics: 2008: IEEE; 2008: 109-114.

25. Saladin L: Pathophysiology of the Motor Systems: Principles and Clinical Presentation. Cerebrovascular disease: stroke 1996:486-512.

26. Ren Y, Kang SH, Park H-S, Wu Y-N, Zhang L-Q: Developing a multi-joint upper limb exoskeleton robot for diagnosis, therapy, and outcome evaluation in neurorehabilitation. IEEE Transactions on Neural Systems and Rehabilitation Engineering 2012, 21(3):490-499.

27. Scholz JP, Schoner G: The uncontrolled manifold concept: identifying control variables for a functional task. Experimental brain research 1999, 126(3):289-306.

28. Verrel J: Distributional properties and variance-stabilizing transformations for measures of uncontrolled manifold effects. Journal of neuroscience methods 2010, 191(2):166-170.

29. Muellbacher W, Richards C, Ziemann U, Wittenberg G, Weltz D, Boroojerdi B, Cohen L, Hallett M: Improving hand function in chronic stroke. Archives of neurology 2002, 59(8):1278-1282.

30. Bard G, Hirschberg GG: Recovery of Voluntary Motion in Upper Extremity Following Hemiplegia. Archives of physical medicine and rehabilitation 1965, 46:567-572.

31. Koh K, Kwon HJ, Kiemel T, Miller RH, Park YS, Kim MJ, Kwon YH, Kim YH, Shim JK: Intra-auditory integration between pitch and loudness in humans: Evidence of super-optimal integration at moderate uncertainty in auditory signals. Scientific reports 2018, 8(1):13708.

32. Koh K, Kwon HJ, Yoon BC, Cho Y, Shin JH, Hahn JO, Miller RH, Kim YH, Shim JK: The role of tactile sensation in online and offline hierarchical control of multi-finger force synergy. Experimental brain research 2015, 233(9):2539-2548.

33. Freitas SM, Duarte M, Latash ML: Two kinematic synergies in voluntary whole-body movements during standing. Journal of neurophysiology 2006, 95(2):636-645.

34. Hsu WL, Scholz JP, Schoner G, Jeka JJ, Kiemel T: Control and estimation of posture during quiet stance depends on multijoint coordination. Journal of neurophysiology 2007, 97(4):3024-3035.

35. McCaskey MA, Wirth B, Schuster-Amft C, de Bruin ED: Dynamic multi-segmental postural control in patients with chronic non-specific low back pain compared to pain-free controls: $A$ cross-sectional study. PloS one 2018, 13(4):e0194512.

36. Latash ML: The bliss (not the problem) of motor abundance (not redundancy). Exp Brain Res 2012, 217(1):1-5.

37. Mattos DJ, Latash ML, Park E, Kuhl J, Scholz JP: Unpredictable elbow joint perturbation during reaching results in multijoint motor equivalence. J Neurophysiol 2011, 106(3):1424-1436.

38. Singh T, Varadhan SK, Zatsiorsky VM, Latash ML: Fatigue and motor redundancy: adaptive increase in finger force variance in multi-finger tasks. J Neurophysiol 2010, 103(6):2990-3000.

39. Zhang W, Scholz JP, Zatsiorsky VM, Latash MLJoN: What do synergies do? Effects of secondary constraints on multidigit synergies in accurate force-production tasks. 2008, 99(2):500-513.

40. Srivastava S, Kao PC, Reisman DS, Higginson JS, Scholz JP: Coordination of muscles to control the footpath during over-ground walking in neurologically intact individuals and stroke survivors. Exp Brain Res 2016, 234(7):1903-1914.

41. Reisman DS, Scholz JP: Workspace location influences joint coordination during reaching in post-stroke hemiparesis. Exp Brain Res 2006, 170(2):265-276. 
42. Butler AJ, Kahn S, Wolf SL, Weiss P: Finger extensor variability in TMS parameters among chronic stroke patients. Journal of neuroengineering and rehabilitation 2005, 2(1):10.

43. Lodha N, Misra G, Coombes SA, Christou EA, Cauraugh JH: Increased force variability in chronic stroke: contributions of force modulation below $1 \mathrm{~Hz}$. PloS one 2013, 8(12):e83468. 


\section{Figures}

Figure 1. A multi-joint intelligent rehabilitation robot, named IntelliArm developed to control the shoulder, elbow and wrist joints simultaneously (a). The shoulder horizontal abduction/adduction, and elbow and wrist flexion/extension are controlled by the shoulder, elbow and wrist motor, respectively. The robotic arm is mounted on a large X-Y table allowing the IntelliArm robot to well-align the mechanical shoulder axis with the horizontal rotation axis of the subject. Schematic diagram that the subject is seated with the upper arm, forearm and hand attached to the IntelliArm (top view in horizontal plane) (b). IntelliArm produces simultaneous movements at shoulder, elbow, and wrist joints, starting to outward direction followed by inward direction. Note that shoulder horizontal adduction, elbow flexion and wrist flexion are positive. For analysis of upper limb coordination, a task equation of end-effector position $\left(\boldsymbol{P}_{\boldsymbol{x}}, \boldsymbol{P}_{\boldsymbol{y}}\right)$ and force $\left(\boldsymbol{F}_{\boldsymbol{x}}, \boldsymbol{F}_{\boldsymbol{y}}\right)$ was expressed as a function of joint angles and torques, respectively.

Figure 2. Joint angles and torques for representative subjects of control (blue) and stroke (red). The mean (solid line) and standard deviation (shaded area) of joint angles and joint torques across trials were presented during outward and inward movements.

Figure 3. Index of coordination (IC) (a), logarithm of task-relevant variability (TRV $V_{\theta}$ (b) and task-irrelevant variability $\left(T I V_{\theta}\right)(\mathrm{c})$ of joint angle were compared between Stroke group (open dot) and Control group (closed dot). There was a significant main effect of Group on both TRV $V_{\theta}$ and $T I V_{\theta}$, indicating Stroke group had a significantly greater both $T R V_{\theta}$ and $T I V_{\theta}$ as compared to Control group. The asterisk indicates a significant difference $(*<0.05)$ between groups. Error bars represent SEM across subjects.

Figure 4. Index of coordination (IC) (a), logarithm of task-relevant variability $\left(T R V_{\tau}\right)(\mathrm{b})$ and task-irrelevant variability $\left(T I V_{\tau}\right)$ (c) of joint angle were compared between Stroke group (open dot) and Control group (closed dot). There was a significant interaction effect Direction $\times$ Group on $I C$. The following pair-wise comparisons revealed that $I C$ in stroke group was a significantly lower during outward direction than inward direction while $I C$ in control group remained unchanged between directions. The decreased IC in Stroke group was main attributed to the increased $T R V_{\tau}$ during outward movement as compared to inward movement. The asterisk indicates a significant difference $(* 0.05)$ between directions in stroke group. Error bars represent SEM across subjects.

Figure 5. Logarithm of task-relevant variability $\left(T R V_{\tau}\right)$ and task-irrelevant variability $\left(T I V_{\tau}\right)$ of joint angle at individual joints during inward and outward directions were compared between Stroke group (open dot) and Control group (closed dot). During inward movement, TRV $\tau$ at elbow joint in Stroke group was significantly greater than in Control group. However, during 
outward movement, $T R V_{\tau}$ at all joints in Stroke group were significantly greater than in Control group. During both directions, $T I V_{\tau}$ at shoulder joint in Stroke group was significantly greater than in Control group. The asterisk indicates a significant difference $(*<0.05 ; * *<0.001)$ between groups. Error bars represent SEM across subjects. 


\section{Figures}

a)

b)

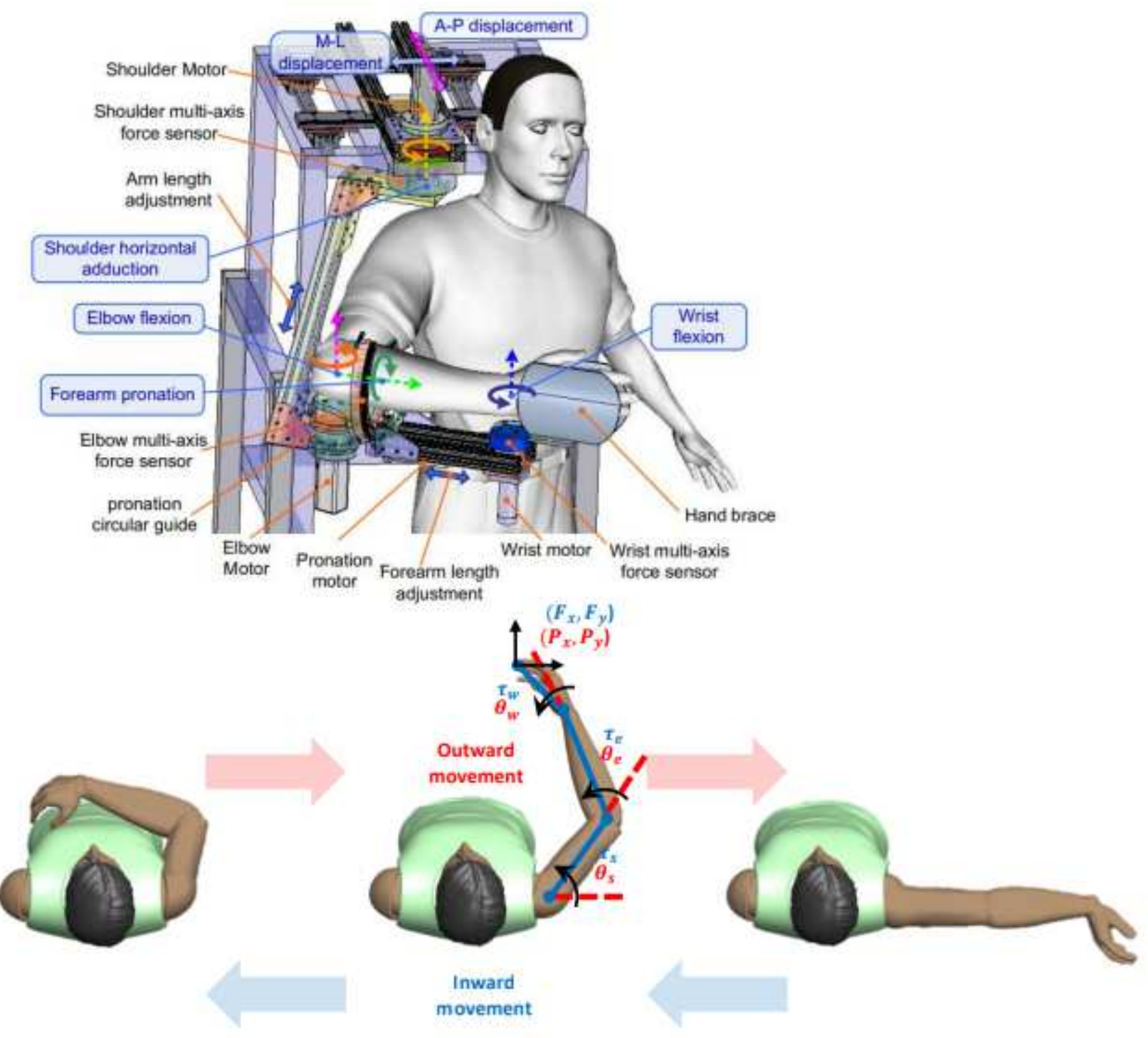

Figure 1

A multi-joint intelligent rehabilitation robot, named IntelliArm developed to control the shoulder, elbow and wrist joints simultaneously (a). The shoulder horizontal abduction/adduction, and elbow and wrist flexion/extension are controlled by the shoulder, elbow and wrist motor, respectively. The robotic arm is mounted on a large $X-Y$ table allowing the IntelliArm robot to well-align the mechanical shoulder axis with the horizontal rotation axis of the subject. Schematic diagram that the subject is seated with the upper arm, forearm and hand attached to the IntelliArm (top view in horizontal plane) (b). IntelliArm produces simultaneous movements at shoulder, elbow, and wrist joints, starting to outward direction followed by inward direction. Note that shoulder horizontal adduction, elbow flexion and wrist flexion are positive. For 
analysis of upper limb coordination, a task equation of end-effector position (P_x,P_y) and force (F_x, $\left.F_{-} y\right)$ was expressed as a function of joint angles and torques, respectively.

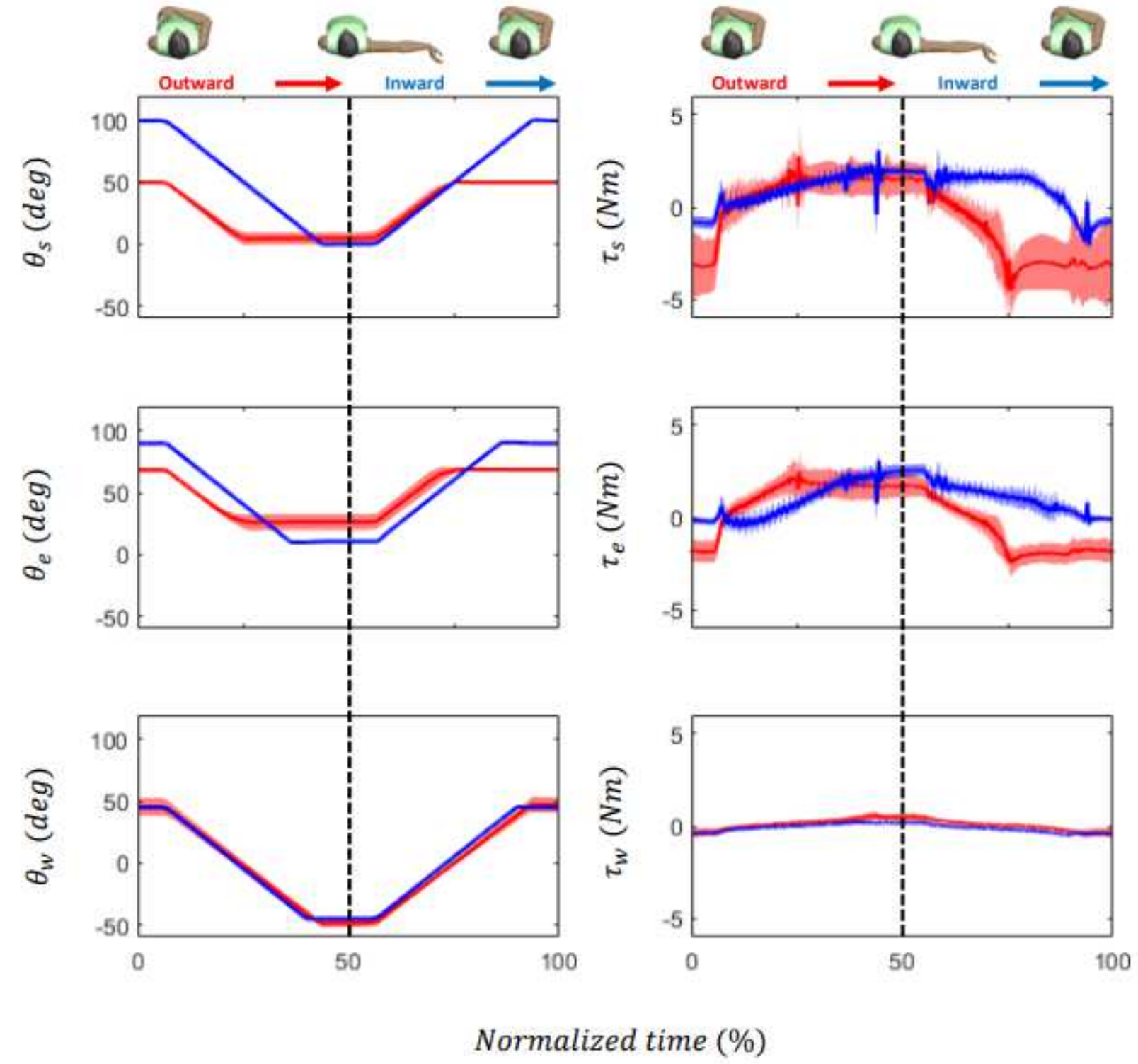

\section{Figure 2}

Joint angles and torques for representative subjects of control (blue) and stroke (red). The mean (solid line) and standard deviation (shaded area) of joint angles and joint torques across trials were presented during outward and inward movements. 
b)

a)
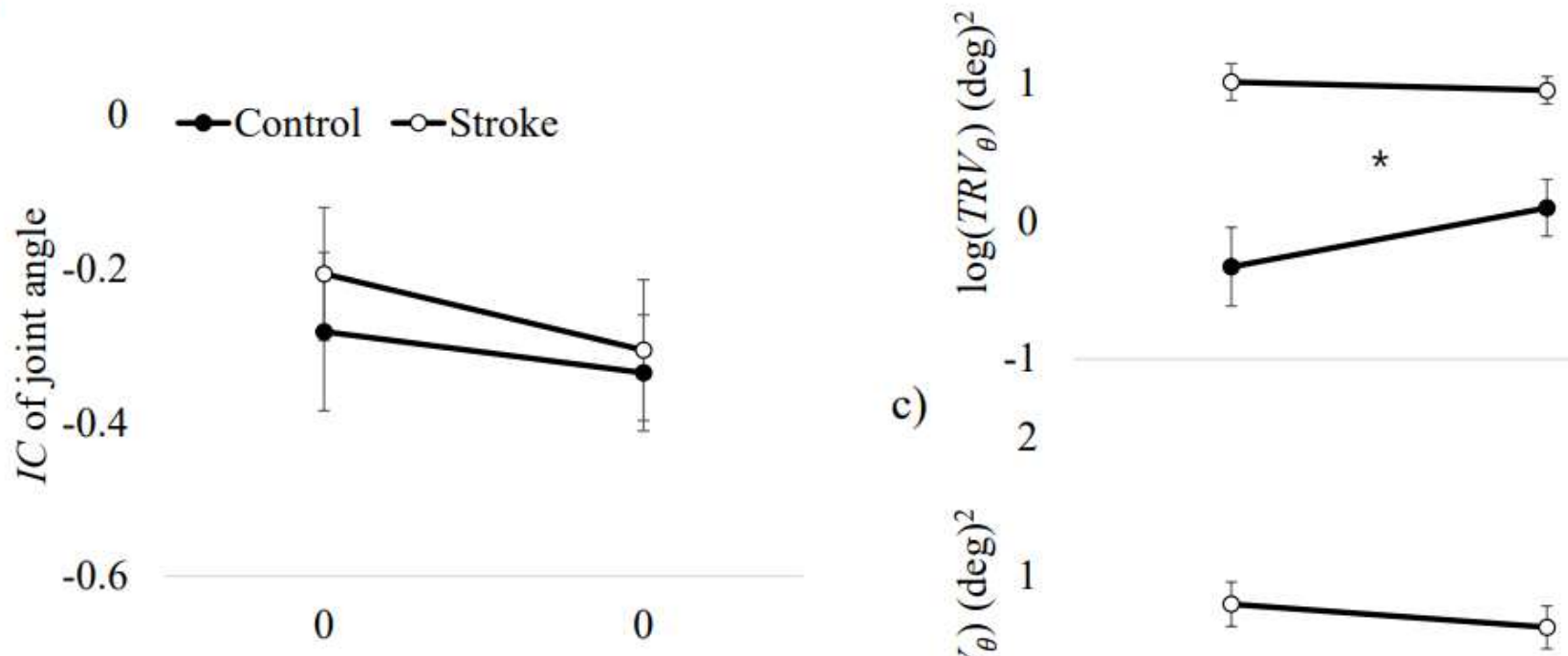

c) $\quad \begin{array}{r}-1 \\ \quad 2\end{array}$
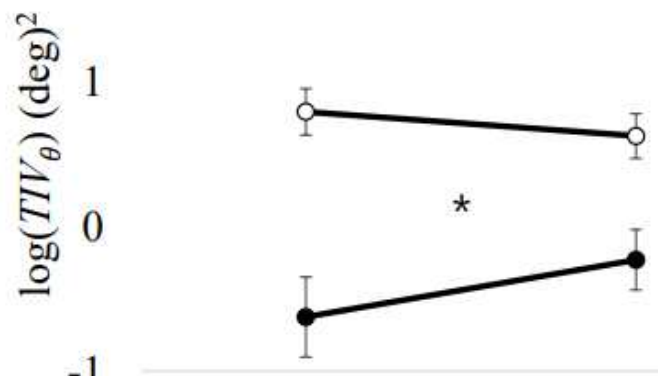

Inward

Outward

Figure 3

Index of coordination (IC) (a), logarithm of task-relevant variability (TRVQ) (b) and task-irrelevant variability (TIVУ) (c) of joint angle were compared between Stroke group (open dot) and Control group

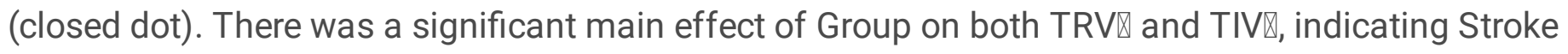

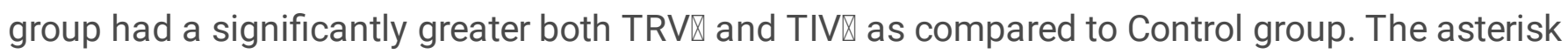
indicates a significant difference $(*<0.05)$ between groups. Error bars represent SEM across subjects. 


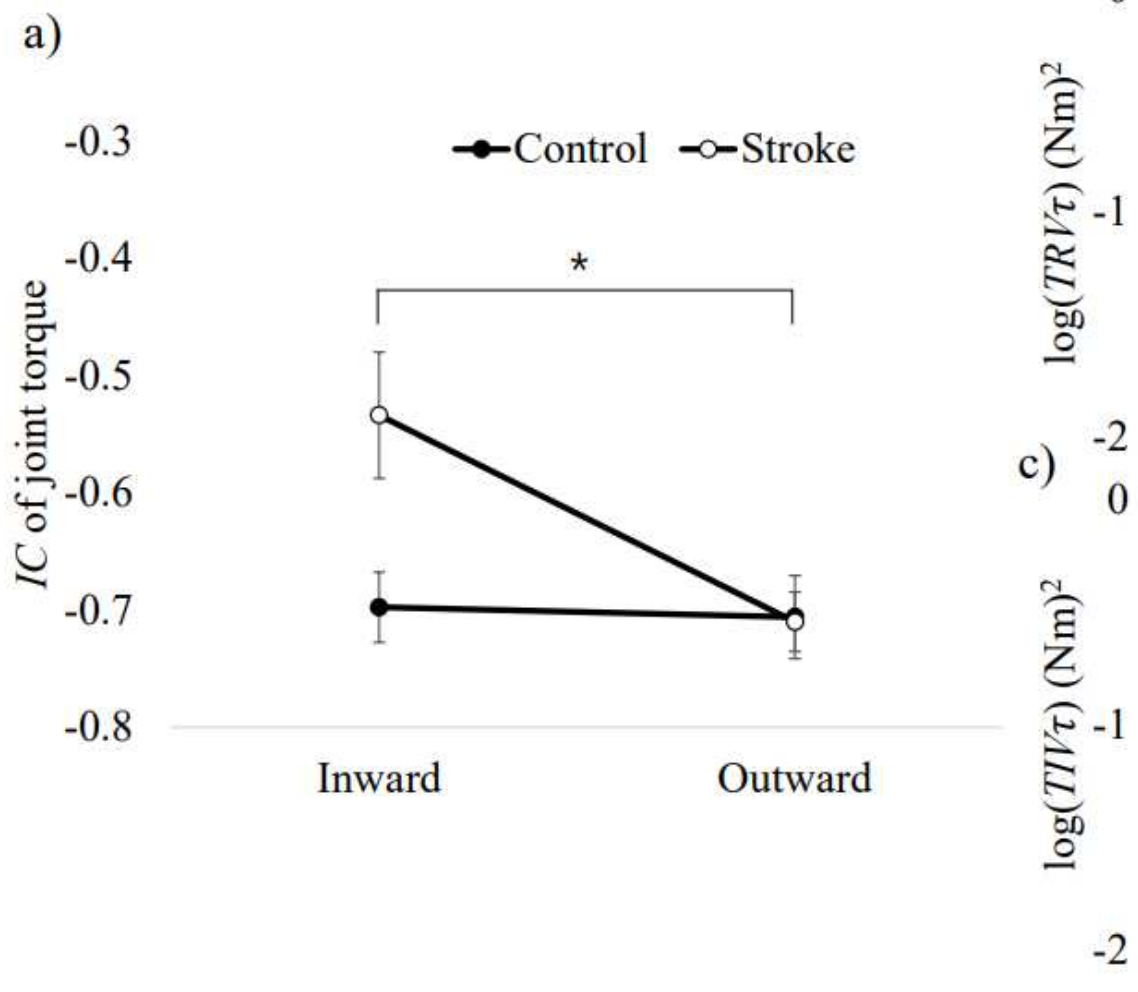

b)
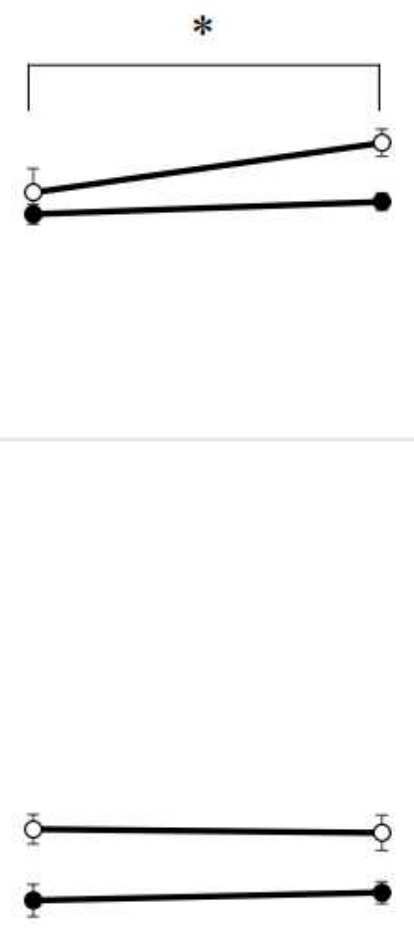

Inward

Outward

Figure 4

Index of coordination (IC) (a), logarithm of task-relevant variability (TRV $\mathbb{\text { }}$ ) (b) and task-irrelevant variability (TIV $\mathbb{Z}$ ) (c) of joint angle were compared between Stroke group (open dot) and Control group (closed dot). There was a significant interaction effect Direction $\times$ Group on IC. The following pair-wise comparisons revealed that IC in stroke group was a significantly lower during outward direction than inward direction while IC in control group remained unchanged between directions. The decreased IC in Stroke group was main attributed to the increased TRV $\Downarrow$ during outward movement as compared to inward movement. The asterisk indicates a significant difference $(*<0.05)$ between directions in stroke group. Error bars represent SEM across subjects. 


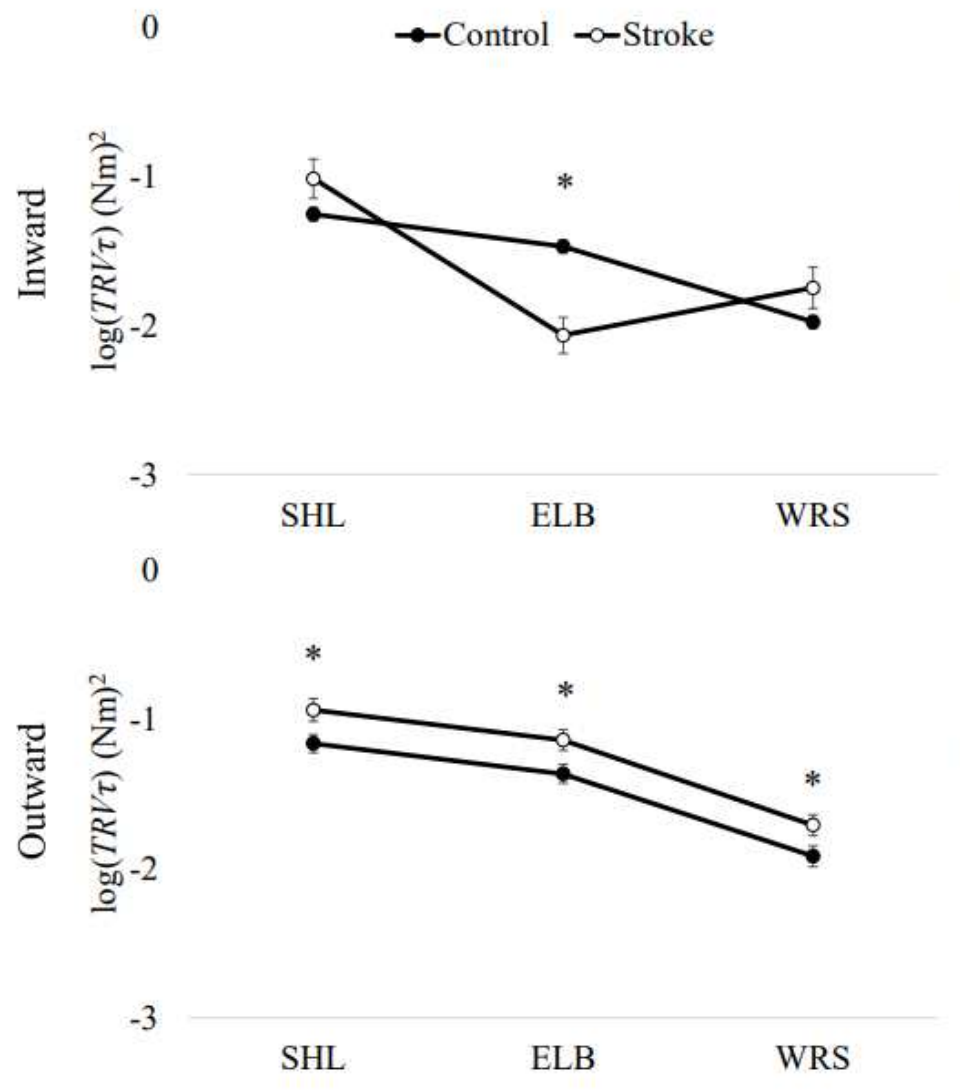

0

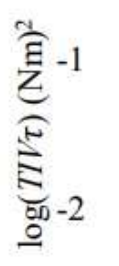

$-3$

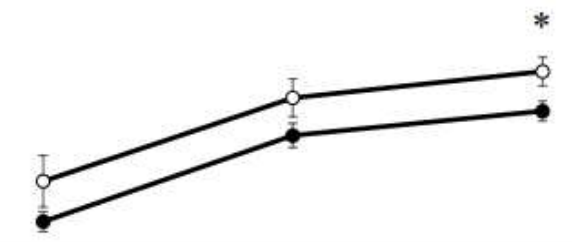

0
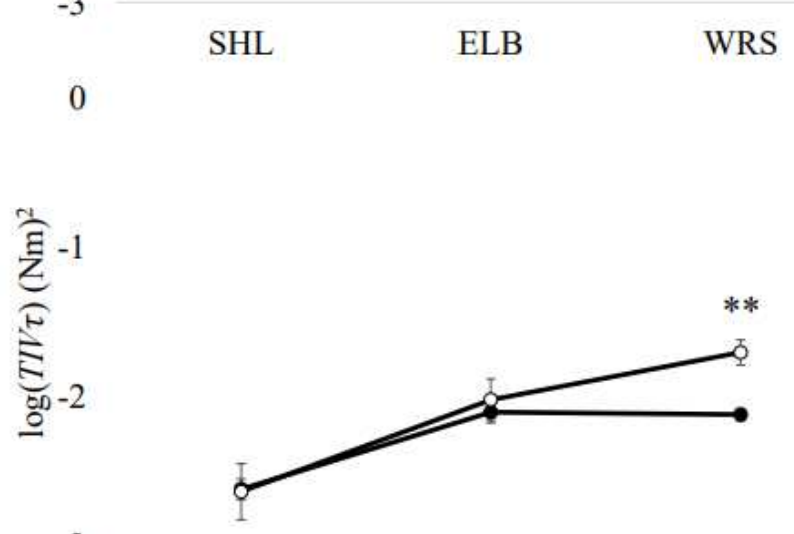

$-3$

SHL

ELB

WRS

\section{Figure 5}

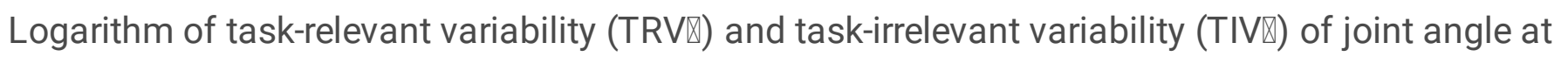
individual joints during inward and outward directions were compared between Stroke group (open dot)

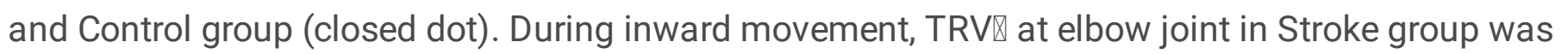

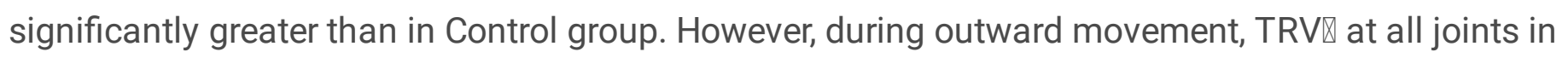

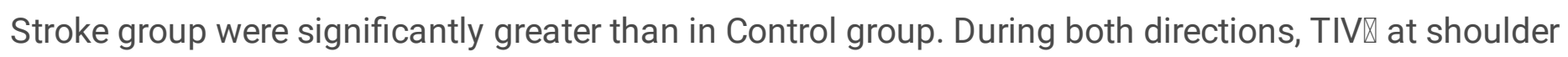
joint in Stroke group was significantly greater than in Control group. The asterisk indicates a significant difference $(*<0.05 ; * *<0.001)$ between groups. Error bars represent SEM across subjects. 